\title{
Escala Fatorial de Socialização- Versão Reduzida: Seleção de Itens e Propriedades Psicométricas
}

\author{
Agreeableness Scale- Short Version: Item Selection \\ and Psychometric Properties
}

\author{
Maiana Farias Oliveira Nunes" ${ }^{*}, a, b, c$, Monalisa Muniz ${ }^{a, d, e}$, \\ Carlos Henrique Sancineto da Silva Nunes ${ }^{a, g}$, Ricardo Primi ${ }^{a}, \&$ Fabiano Koich Miguel ${ }^{a, d, f}$ \\ ${ }^{a}$ Universidade São Francisco, ${ }^{b}$ Universidade Federal do Rio Grande do Sul, ${ }^{c}$ Faculdade Avantis, \\ ${ }^{d}$ Faculdade Integradas Einstein de Limeira, ${ }^{e}$ Faculdades de Tecnologia, Ciências e Educação (FATECE), \\ ${ }^{f}$ Universidade Estadual de Londrina \& ${ }^{8}$ Universidade Federal de Santa Catarina
}

\begin{abstract}
Resumo
O objetivo desse estudo foi selecionar itens da Escala Fatorial de Socialização (EFS) para a obtenção de uma versão reduzida, que mantivesse propriedades psicométricas adequadas. Baseou-se em uma amostra de 1.100 sujeitos. Para a seleção de itens, realizou-se análise qualitativa, buscando aqueles sem conteúdo clínico explícito e uma análise quantitativa, pelo modelo de Rasch. Tais critérios permitiram reduzir a EFS de 70 para 28 itens. As características psicométricas da versão reduzida foram verificadas pela comparação entre versões por Rasch e pela reanálise dos dados de estudos de validade realizados com a EFS. A versão reduzida manteve características psicométricas adequadas, o que sugere a possibilidade de utilização dessa versão da EFS em situações de avaliação com tempo restrito.

Palavras-chave: Personalidade; Avaliação psicológica; Teoria de resposta ao item; Psicometria; Medidas da personalidade.
\end{abstract}

\begin{abstract}
This study aimed at selecting items from the Agreeableness Factor Scale for obtaining a short version of this test that could keep adequate psychometric properties. One thousand one hundred participants composed the sample. Items were selected using a qualitative strategy, which focused on item content that was not related to clinical descriptions and a quantitative analysis based on Rasch's model. The scale was reduced from 70 to 28 items, based on these criteria. In order to check the psychometric properties of the short version, both versions were compared by Rasch indices and by reanalyzing validity studies conducted with the original scale. The short version kept good psychometric properties, which suggests the possibility of using it when there is time restriction.

Keywords: Personality; Psychological assessment; Item response theory; Psychometrics; Personality measures.
\end{abstract}

As pesquisas sobre avaliação da personalidade, a partir da década de 1960 (Tupes \& Christal, 1961/1992), têm convergido para um modelo atualizado da teoria de traços da personalidade (Allport, 1961), denominado de Cinco Grandes Fatores. A estrutura da personalidade, no Modelo dos Cinco Grandes Fatores (CGF), pode ser compreendida por cinco dimensões amplas, denominadas Neuroticismo (Emotional Stability ou Neuroticism), Socialização (Agreeableness), Extroversão (Extroversion ou Surgency), Realização (Conscientious-

\footnotetext{
* Endereço para correspondência: Universidade São Francisco, Programa de Pós-Graduação Stricto Sensu em Psicologia, Rua Alexandre Rodrigues Barbosa, 45, Centro, Itatiba, SP, Brasil, CEP 13251-900. E-mail: maiananunes@mac.com

Financiamento CAPES, CNPq e FAPESP.
}

ness) e Abertura para novas experiências (Openness ou Culture). No Brasil, o modelo tem sido investigado a partir do final da década de 1990 (exemplos Ávila \& Stein, 2006; Bueno, Oliveira, \& Oliveira, 2006; Hutz \& Nunes, 2001; Hutz et al., 1998; Maciel \& Yoshida, 2006; Nunes \& Hutz, 2007a, 2007b, 2007c; Nunes, Hutz, \& Nunes, 2010; Nunes et al., 2008; Santos, Sisto, \& Martins, 2003).

No modelo dos CGF, Socialização é uma dimensão interpessoal que se refere aos tipos de interações que uma pessoa apresenta ao longo de um contínuo que se estende da compaixão ao antagonismo. Pessoas que são altas em Socialização tendem a se mostrar generosas, bondosas, afáveis, prestativas e altruístas. Indivíduos com esse perfil tendem a querer ajudar os outros, a ser responsivos e empáticos, e acreditam que a maioria das outras pessoas irá agir da mesma forma. Por outro lado, pessoas 
com baixos escores no fator tendem a ser cínicas, vingativas, não cooperativas e irritáveis, podendo apresentar também reações de auto e hetero-agressividade (P. T. Costa \& Widiger, 2002). Na mesma direção, P. T. Costa e McCrae (1992) indicam que Socialização envolve aspectos como perceber os outros como bem intencionados; ser franco e direto; pré-disposição para ajudar os outros; demonstração de deferência em situações de conflito; humildade; atitudes de simpatia, compaixão e preocupação pelo lado humano das políticas sociais.

Por sua vez, Widiger, Trull, Clarkin, Sanderson e Costa (2002) apontam para a importância do fator Socialização para a avaliação de alguns distúrbios psiquiátricos. Assim, escores baixos em Socialização são associados ao Transtorno Paranóide, ao Transtorno AntiSocial, Narcisista e Borderline. Por outro lado, escores altos em Socialização estão relacionados aos Transtornos de Personalidade Histriônica e Dependente. Desse modo, pode-se destacar a relevância da avaliação dessa dimensão da personalidade no âmbito clínico.

No contexto organizacional, o fator Socialização foi estudado por Witt, Kacmar, Carlson e Zivnuska (2002), que avaliaram a personalidade de 540 supervisores e respectivos subordinados de uma empresa privada. Entre os resultados, Socialização foi a dimensão que interagiu mais significativamente na previsão do desempenho. Essa mesma dimensão correlacionou-se positiva e significativamente com uma medida de facilitação interpessoal. Desse modo, os autores destacaram que é necessário considerar tanto a situação em que se pretende prever um comportamento, como os antecedentes de personalidade.

Em seu estudo, Barrick e Mount (1991) demonstraram que Socialização relaciona-se positivamente com a performance no trabalho. De maneira semelhante, Ross, Rausch e Canada (2003) observaram que esse fator encontra-se associado a atitudes orientadas para o sucesso no trabalho, assim como para posturas de maior cooperação e menos competição. Esses dados indicam que altos escores de Socialização no âmbito das empresas podem sugerir uma pré-disposição a se comportar de maneira cooperativa e disponível. De modo semelhante, Socialização também tem demonstrado utilidade no contexto da Orientação Vocacional/Profissional. A título de ilustração, a pesquisa de Pérez, Cupani, e Beltramino (2004) verificou que o fator Socialização possui utilidade preditiva para as intenções de escolha de carreira, principalmente nas áreas de Tecnologia e Ciências Humanas. Desse modo, percebe-se o potencial de estudo do construto Socialização em diversas áreas de pesquisa em Psicologia, sendo, portanto, relevante a disponibilização de instrumentos para a avaliação do mesmo.

No Brasil, há um teste, denominado "Escala Fatorial de Socialização" ([EFS], Nunes \& Hutz, 2007b) para a avaliação específica desse construto, que possui parecer favorável do Conselho Federal de Psicologia ([CFP], 2007). Nacionalmente, outras escalas objetivas para avaliação da personalidade de adultos encontram-se aprovadas pelo CFP, algumas delas baseadas em outros modelos teóricos. Entre estas, aquelas que avaliam traços comparáveis à Socialização e que são comercializadas são a Escala de Personalidade de Comrey ([CPS], F. R. Costa, 2003), o Inventário Fatorial de Personalidade ([IFP], Pasquali, Azevedo, \& Ghesti, 1997) e o QUATI (Zacharias, 2003). Esses baseiam-se, respectivamente, nas teorias de personalidade de Comrey, Murray e Jung, que compreendem a avaliação da personalidade por meio de uma quantidade distinta de fatores, além de possuírem, individualmente, escalas com mais de 90 itens. Essa quantidade de itens exige maior dispêndio de tempo para que um teste seja respondido, o que pode dificultar ou impossibilitar a realização de coletas de dados em certos contextos.

Considerando a questão do tamanho das escalas e o tempo necessário para respondê-las, formas mais breves de testes têm sido pesquisadas internacionalmente para a avaliação de personalidade. Gosling, Rentfrow e Swann (2003) destacam a utilidade de medidas breves de avaliação da personalidade em situações em que os participantes precisam se avaliar e repetir o mesmo processo com outros, pesquisas de larga escala e avaliações longitudinais. Esses autores relatam dois estudos com escalas de cinco e dez itens para medir os CGF. Uma versão mais ampla foi utilizada (com os descritores de Goldberg, 1982), que constava de 100 itens, para a comparação com as versões reduzidas. Entre as formas reduzidas, aquela que apresentou melhores características psicométricas foi a de 10 itens. Os autores destacam, contudo, as limitações de escalas muito breves, entre elas a fraca qualidade psicométrica, em comparação com escalas maiores e a impossibilidade de avaliar as sub-dimensões dos fatores. Os principais benefícios apontados com este tipo de medida são a extensão do escopo em que o CGF pode ser utilizado e a eliminação da redundância de itens, que pode gerar a frustração e tédio nos respondentes.

Na mesma direção, Reise e Henson (2000) avaliaram as propriedades do NEO-PI-R por meio da Teoria de Resposta ao Item, com uma amostra de 1.059 estudantes universitários. Esses dados foram usados para simular uma seleção de itens para uma testagem adaptativa do teste. Entre os resultados encontrados, os autores destacaram que, para as 30 facetas, apenas 3 ou 4 itens seriam necessários para se obter resultados adequados, e não os 8 itens por faceta, conforme a versão originalmente proposta pelos autores do teste. Ainda, foi possível uma redução do teste sem perder a qualidade da sua precisão, ao comparar versões do teste com quantidades diferentes de itens por faceta. Os autores discutiram que a versão adaptativa pode ser tão boa quanto a versão não adaptativa com 4 itens, desde que os melhores 4 itens de cada faceta sejam escolhidos. Como critérios para a seleção dos melhores itens, os autores basearam-se em 3 aspectos: altas correlações item-total, altas cargas fatoriais e elevados valores de discriminação obtidos na 
análise de TRI. Um bom item, nessa perspectiva, é útil numa extensão razoavelmente grande do traço latente. Mais especificamente, os autores indicam que apenas 4 itens para cada faceta foram capazes de recuperar a mesma informação sobre o traço latente e com erros-padrões de theta semelhantes, quando comparados com a versão com 8 itens.

Uma vez que a Teoria de Resposta ao Item (TRI) foi um dos recursos utilizados no presente artigo, faz-se necessário um detalhamento sobre alguns pressupostos da mesma. A TRI é realizada por meio de modelos matemáticos que buscam explicar ou descrever a relação entre a resposta observada de uma pessoa em um dado teste e o traço latente correspondente (construto medido). Os parâmetros investigados nessa teoria são a discriminação (a), dificuldade (b) e acerto ao acaso (c). Apenas o parâmetro da dificuldade foi selecionado como uma das análises desse artigo, referindo-se ao modelo de Rasch, de modo que se torna necessário uma definição do mesmo. A dificuldade do item informa o local (a região de theta) onde o item funciona melhor ao longo da escala de habilidade, sendo que esse parâmetro indica o valor de theta correspondente a 50\% de chance de acertar o item. Esse pressuposto leva a algumas consequiências, a saber, para pessoas com menor habilidade, há uma menor probabilidade de acerto a itens difíceis, ou seja, é necessário um maior nível de habilidade para acertar o item. Por outro lado, itens com menor nível de dificuldade (mais fáceis) podem ser acertados tanto por pessoas com thetas maiores como por pessoas com thetas menores (Embretson \& Reise, 2000).

Ainda de acordo com esses autores, para que um teste de personalidade seja usado em uma população com diferentes níveis de traço latente, é importante que o nível de dificuldade dos itens tenha uma extensão suficiente para "cobrir" a amplitude planejada no construto em questão. Assim, podem-se usar mapas de itens e pessoas, que ajudam a fazer uma observação sobre quais áreas de theta já possuem muitos itens e quais delas apresentam lacunas. Outro método útil para a seleção de itens é a correlação item-theta, que indica o grau de associação entre itens, considerados individualmente, com as diferentes magnitudes de theta. Essa análise é semelhante à correlação item-total realizada pela Análise Clássica de itens, porém com a vantagem da correlação ser feita com o traço latente, e não com o escore bruto, como é o caso nas análises clássicas (Embretson \& Reise, 2000).

Outra estratégia que pode ser usada para a seleção de itens é a análise de conteúdo dos mesmos, que parte do pressuposto que alguns itens/comportamentos podem ser mais ou menos relacionados diretamente com as inferências que se deseja fazer. Assim, pode-se buscar compreender a relevância e representatividade dos itens quando observados individualmente, de acordo com os diferentes propósitos selecionados. Pode-se delinear diferentes formas do mesmo teste para diferentes propósitos, em função dos objetivos da testagem, por meio da análise de conteúdo. A mesma realiza-se, de modo geral, por meio do consenso entre especialistas na área, que avaliam a pertinência e representatividade dos itens para o objetivo em questão. Esse recurso de análise pode complementar abordagens quantitativas de seleção de itens, buscando uma compreensão embasada no referencial teórico que orienta as escolhas dos especialistas (Urbina, 2007).

Considerando a relevância da avaliação do fator Socialização nos diferentes contextos, é importante planejar a disponibilização de instrumentos de avaliação mais reduzidos, para beneficiar os psicólogos ou pesquisadores que buscam uma medida mais simples e rápida do construto. Conforme já mencionado, a EFS é a única escala aprovada pelo CFP para avaliação dessa dimensão da personalidade com o referencial teórico dos Cinco Grandes Fatores (CGF), sugerindo a utilidade da criação de outras formas deste teste, buscando atingir públicos variados e em condições em que a aplicação da EFS torna-se difícil por falta de tempo.

Os objetivos deste estudo envolveram a seleção de itens da EFS para a construção de uma versão reduzida da mesma e a verificação de algumas propriedades psicométricas. Mais especificamente, buscou-se comparar, por meio da Análise de Rasch, as propriedades psicométricas das duas versões da escala - original e reduzida - e reanalisar dados de dois estudos de validade de critério, considerando apenas os itens da versão reduzida da escala. Essa proposta torna-se relevante pelo tamanho original da EFS, que pode limitar seu uso em situações em que o psicólogo dispõe de pouco tempo para a avaliação. Esse estudo pode ser visto como uma continuação da pesquisa de Nunes e Hutz (2007c), que descreveu algumas propriedades psicométricas da versão de 70 itens da EFS.

\section{Método}

\section{Participantes}

A amostra foi composta por 1.100 sujeitos, sendo $70,1 \%$ mulheres, com idade variando de 14 a 64 anos $(M=21,38$, $D P=5,84)$. Sobre a escolaridade, foi possível obter essa informação de 745 participantes. A escolaridade dos informantes $(N=745)$ foi variada, porém houve maior participação de estudantes secundaristas $(40,7 \%)$ e os demais eram universitários $(59,3 \%)$, com maior participação de alunos do curso de psicologia. Os dados foram coletados na Bahia (41,9\%), Rio Grande do Sul $(26,1 \%)$, Paraíba (17, 0\%), São Paulo $(14,1 \%)$ e Santa Catarina $(0,8 \%)$. Vale destacar que essa é a mesma amostra descrita nos estudos que analisaram a estrutura interna da EFS (Nunes, 2005; Nunes \& Hutz, 2007c).

\section{Instrumento}

O presente estudo utilizou a Escala Fatorial de Socialização ([EFS], Nunes \& Hutz, 2007b), que é um instrumento objetivo para avaliação de um componente da personalidade a partir do modelo dos CGF (P. T. Costa 
\& McCrae, 1992; Digman, 2002; McCrae \& John, 1992; Widiger et al., 2002). A EFS é composta por 70 itens de auto-relato que descrevem sentimentos, atitudes e opiniões, a partir de assertivas, apresentadas numa escala Likert de 7 pontos, em que os participantes indicam quão bem cada frase os descreve.

A EFS é composta por três subescalas, denominadas Amabilidade (S1), Pró-sociabilidade (S2) e Confiança nas pessoas (S3). A dimensão S1 indica quão atenciosas, gentis e empáticas as pessoas procuram ser com os outros; S2 engloba os comportamentos de risco, confronto ou adesão às normas sociais, auto e hetero-agressividade e padrões de consumo de bebidas alcoólicas; e, por fim, S3 agrupa itens que avaliam quanto o sujeito confia nos outros e acredita que os outros poderão prejudicá-lo.

Os estudos de validade da EFS envolveram a análise da estrutura interna e a validade de critério. Para verificação da última, foram comparados os resultados de pessoas em tratamento de dependência química com universitários e com a amostra normativa, tendo encontrado diferenças significativas para todas as sub-escalas do teste, especialmente para Pró-sociabilidade (Nunes \& Hutz, 2007b; Nunes, Nunes, \& Hutz, 2006; Nunes, Nunes, Cunha, \& Hutz, 2009). Para a seleção de itens por meio da análise fatorial, foram considerados os autovalores, a correlação item-total, o scree plot e as cargas fatoriais dos itens nas facetas identificadas. Quanto à precisão, o alfa de Cronbach foi de 0,91, 0,84 e 0,80 para as facetas Amabilidade (S1), Pró-sociabilidade (S2) e Confiança nas pessoas (S3), respectivamente. A escala como um todo apresentou uma consistência de 0,92 (Nunes \& Hutz, 2007b).

\section{Procedimentos de Coleta}

Com relação à aplicação da EFS, os participantes foram voluntários e responderam a escala mediante Termo de Consentimento Livre e Esclarecido. A aplicação foi feita de forma coletiva, por estudantes de psicologia ou psicólogos treinados para realizarem essa coleta.

\section{Resultados e Discussão}

Os objetivos desse trabalho foram selecionar itens para uma versão reduzida da EFS e verificar algumas propriedades psicométricas dessa. No que diz respeito aos procedimentos adotados para a redução da escala, foram feitas conjuntamente análises quantitativas e qualitativas. Inicialmente serão descritos os procedimentos para a seleção de itens, seguidos pela análise de Rasch para a comparação das duas versões da escala e a reanálise de dois estudos de validade de critério realizados com a EFS.

$\mathrm{Na}$ análise qualitativa realizada para a seleção de itens, seguindo as recomendações de Urbina (2007), foram verificados os conteúdos dos itens, que foram julgados por seis psicólogos, sendo 1 pós-doutorando, 1 doutor e 4 doutorandos, todos pesquisadores da área de Avaliação
Psicológica. Nessa etapa foram priorizados itens que não enfocassem conteúdo clínico, sendo essa opção feita com o objetivo de fornecer uma escala que pudesse ser utilizada em contextos mais gerais, como em seleção de pessoal ou pesquisa, e não necessariamente para auxiliar na avaliação de características psicopatológicas. Desse modo, buscou-se selecionar itens com conteúdo apropriado a objetivos mais gerais.

A análise que se seguiu para a seleção dos itens foi realizada pelo modelo de Rasch, com escalas graduadas/ créditos parciais (Wright \& Masters, 1982), com a utilização do programa Winsteps. Vale destacar que mesmo realizando uma análise qualitativa prévia dos itens que comporiam a escala reduzida, optou-se por analisar quantitativamente todos os 70 itens da EFS para que se pudesse verificar, juntamente com as propriedades psicométricas, se a seleção qualitativa se sustentava. Assim, a seleção de itens para a versão reduzida foi feita apenas após a comparação da análise qualitativa e da de Rasch, e não de uma das duas estratégias de análise considerada isoladamente.

Para a seleção de itens pela análise de Rasch, foram verificados os parâmetros de dificuldade dos itens, correlação item-theta e índices de ajuste (infit e outfit) dos itens. A dificuldade dos itens, no modelo de Rasch, é associada ao nível de habilidade no qual as pessoas têm $50 \%$ de chance de acerto, sendo que pode variar entre -4 e +4. Ao considerar itens sobre personalidade, o parâmetro dificuldade deve ser entendido em termos da magnitude do traço latente das pessoas, e, conseqüentemente, não em termos de acerto e erro, mas de adesão ao conteúdo dos itens. Vale destacar que, na TRI, a dificuldade dos itens e o nível de habilidade das pessoas é quantificado na mesma escala, que segue uma métrica semelhante ao escore $\mathrm{z}$, e que estas informações podem ser diretamente comparadas para usos específicos. Já o parâmetro Outfit refere-se à média dos ajustes dos itens que é mais sensível aos outliers, em que o desajuste do item ou sua discrepância ocorre distante do nível de habilidade/nível do traço do sujeito, ou seja, a pessoa não adere a itens muito fáceis para sua habilidade ou adere ao conteúdo de itens muito distantes da sua habilidade. Infit também é uma medida de desajuste do item, em que há discrepâncias próximas do nível de habilidade do sujeito, ou seja, ele não acerta/não adere a itens próximos do seu nível de habilidade (Baker, 2001).

Como critérios para seleção dos itens, a correlação itemtheta buscada foi o mais próximo de 1 e o theta deveria abranger diferentes áreas da escala. Em outras palavras, na análise da comparação da dificuldade dos itens com a habilidade das pessoas, foram privilegiados itens que fossem úteis para pessoas com níveis variados do traço latente, desde pessoas com baixo nível de socialização, passando por níveis médios e chegando aos altos. Ressalta-se que o item, para ser considerado ajustado, deveria apresentar índices de infit e outfit de no máximo de 
1,4 , critério que segue as recomendações de Wright e Stone (2004).

Quanto à dificuldade dos itens, essa variou entre $-0,33$ e 0,65 , indicando que os itens possuíam uma dificuldade média, ou seja, próximos de zero. Com essa informação, buscou-se incluir itens que estivessem representando diferentes áreas dentro da extensão de theta medida pela EFS, ou seja, entre -0,33 e 0,65. Com relação aos índices de ajustes dos itens, infit e outfit, apenas 17 itens dos 70 demonstraram altos níveis de desajustes, considerando um desses dois parâmetros. Por sua vez, sete itens apresentaram correlação item-theta inferiores a 0,30 , o que foi considerado como um patamar não desejado para o item ser mantido. Desse modo, não foram selecionados para a versão reduzida da escala os itens com desajuste em infit ou outfit ou nos casos que a correlação itemtheta encontrou-se em patamar fora do esperado.

Por fim, outro critério usado para a seleção de itens foi que houvesse uma quantidade relativamente equilibrada de itens para cada faceta da EFS, de modo que nenhuma delas fosse sub-representada na versão reduzida. Do total de 70 itens, 28 foram mantidos, sendo 11 da Escala de Amabilidade, 9 de Pró-Sociabilidade e 8 de Confiança nas pessoas. Desse modo, o critério mínimo sugerido por Reise e Henson (2000) de 3 ou 4 itens por faceta foi alcançado. Tendo realizado a seleção dos itens, procederam-se as análises para verificar a equivalência entre a versão reduzida e a completa da EFS. A Tabela 1 apresenta uma comparação dos resultados dos parâmetros de Rasch com as versões com 70 e 28 itens.

Tabela 1

Propriedades Psicométricas Obtidas pela Análise de Rasch das Respostas das Pessoas à EFS com as Versões de 70 e 28 Itens

\begin{tabular}{lcccccccc}
\hline & \multicolumn{2}{c}{ Escore bruto } & \multicolumn{2}{c}{ Theta } & \multicolumn{2}{c}{ INFIT } & \multicolumn{2}{c}{ OUTFIT } \\
\cline { 2 - 8 } & 70 itens & 28 itens & 70 itens & 28 itens & 70 itens & 28 itens & 70 itens & 28 itens \\
\hline Média & 384,0 & 150,9 & 0,52 & 0,50 & 1,09 & 1,07 & 1,06 & 1,04 \\
DP. & 47,3 & 20,4 & 0,32 & 0,36 & 0,47 & 0,53 & 0,50 & 0,55 \\
Máximo & 479,0 & 192,0 & 2,07 & 2,26 & 3,88 & 3,56 & 5,12 & 4,33 \\
Mínimo & 151,0 & 58,0 & $-0,58$ & $-0,71$ & 0,25 & 0,16 & 0,25 & 0,15 \\
\hline
\end{tabular}

Como é possível observar, as médias e desvios-padrão do theta foram muito semelhantes, indicando a equivalência entre as duas formas da escala para medir o traço latente Socialização, conforme proposto no modelo dos Cinco Grandes Fatores. Quanto aos índices de ajuste das escalas, infit e outfit, verificaram-se padrões equivalentes de média e desvio-padrão, sugerindo que a redução da escala não prejudicou esses parâmetros, mantendo-se dentro dos patamares recomendados, com valores próximos de 1 (Wright \& Stone, 2004). Assim, do mesmo modo que outras pesquisas (Reise \& Henson, 2000), foi verificada a possibilidade de redução da escala sem perder as características favoráveis observadas com a escala completa.

Outro aspecto considerado para comparar a versão reduzida e a completa foram os índices de consistência interna, mais especificamente o alfa de Cronbach e a Separação. O parâmetro separação considera a variância das estimativas de pessoas testadas em certos grupos, relacionando o erro de medida de cada pessoa, ou seja, informa sobre quão confiável pode ser a separação de pessoas em grupos, com base em seus escores. Dito de outro modo, envolve a análise da proporção de variância verdadeira, considerando o erro padrão de medida. Quanto maior a separação, mais precisa é a medida. Um índice de separação de 2 é equivalente a um alfa de Cronbach de 0,80 , sendo considerado um patamar adequado (Wright \& Stone, 2004). O programa Winsteps fornece os parâmetros de consistência interna real e do modelo. No primeiro caso, são expostos os resultados observados e, no segundo, os dados ajustados ou modelados, em que o programa busca a melhor solução estatística possível, com base nos dados empíricos. A Tabela 2 apresenta essas informações.

Tabela 2

Consistência Interna da EFS com as Versões de 70 e 28 Itens, pela Análise de Rasch

\begin{tabular}{lcc}
\hline \multicolumn{1}{c}{ Parâmetros } & 70 itens & 28 itens \\
\hline Separação Real & 2,93 & 1,94 \\
Separação do Modelo & 3,40 & 2,28 \\
Alfa de Cronbach Real & 0,90 & 0,79 \\
Alfa de Cronbach do Modelo & 0,92 & 0,84 \\
\hline
\end{tabular}

De acordo com a Tabela 2, verificou-se uma diminuição da consistência interna como um todo, seja analisando a Separação ou a o alfa de Cronbach, porém isso é justificável, uma vez que a precisão de um teste é dependente do número de itens. De todo modo, o alfa de Cronbach com a versão reduzida manteve-se em um 
patamar adequado (Pasquali, 2003), de aproximadamente de 0,80 , do mesmo modo que o índice de separação, segundo as recomendações de Wright e Stone (2004).

Foi realizada ainda uma análise da correlação entre o theta das pessoas na versão com 28 itens em comparação com o theta na versão de 70 itens. A correlação entre o theta nas duas versões foi de $0,93, \operatorname{com} p<0,001$. O fato de essa correlação ter sido significativamente alta e positiva corrobora as análises comparativas expostas anteriormente, apontando que, mesmo com a redução da escala, essa se manteve psicometricamente equivalentes à escala original. De modo geral, os resultados da comparação das versões por meio das análises de Rasch sugerem que foi possível obter uma medida adequada do fator Socialização com a versão reduzida, do mesmo modo que foi verificado em versões reduzidas de avaliação da personalidade nos estudos de Gosling et al. (2003) e Reise e Henson (2000).

Por fim, após a realização da comparação da versão reduzida com a completa por meio da análise de Rasch, procedeu-se a reanálise de dois estudos de critério da EFS (Nunes, Nunes, et al., 2009; Nunes et al., 2006), presentemente considerando a versão reduzida (28 itens). A pesquisa de Nunes, Nunes, et al. (2009) analisou se pessoas adictas a substâncias psicoativas possuíam níveis diferenciados do traço Socialização. Para tanto, fo- ram analisados os dados de dois grupos, sendo um deles formado por 54 clientes de uma clínica para tratamento de dependência química (grupo clínico) e outro de 35 estudantes universitários. Adicionalmente, os dados desse estudo foram comparados com algumas informações da amostra de normatização da EFS.

Nesse estudo foi feita a comparação dos resultados obtidos pelos participantes com os da amostra normativa da EFS, com a utilização de teste $t$ de Student (Nunes, Nunes, et al., 2009). A Tabela 3 compara os resultados da análise de diferenças de média do grupo clínico com a amostra normativa, considerando a EFS nas versões completa (70 itens) e reduzida (28 itens).

$\mathrm{O}$ estudo com a escala original havia identificado diferenças significativas em todas as facetas da EFS e no escore total. Do mesmo modo, presentemente observouse que a escala reduzida também diferenciou os participantes do grupo clínico, em comparação com a amostra normativa, apesar dos valores de $t$ terem diminuído. Outra análise foi realizada (Nunes, Nunes, et al., 2009) para verificar se os resultados da EFS eram capazes de prever em que grupo os participantes do estudo estavam alocados (universitários ou grupo clínico). Para tanto, foi realizada uma regressão logística. Para o presente artigo, foi reanalisada apenas a capacidade preditiva do modelo, que é exposta na Tabela 4.

Tabela 3

Comparação de Médias na EFS com as Versões de 70 e 28 Itens, entre a Amostra Normativa e o Grupo Clínico

\begin{tabular}{llcccc}
\hline \multicolumn{2}{l}{ Versão da EFS } & Média & Desvio padrão & $t$ & $p$ \\
\hline 70 itens & S1 & $-0,75$ & 1,30 & 4,66 & $<0,001$ \\
& S2 & $-1,08$ & 1,24 & 6,73 & $<0,001$ \\
& S3 & $-0,53$ & 1,02 & 3,33 & $<0,001$ \\
& Escore total & $-1,00$ & 1,21 & 6,23 & $<0,001$ \\
& S1 & $-0,43$ & 1,10 & 2,69 & $<0,001$ \\
& S2 & $-0,73$ & 1,24 & 4,54 & $<0,001$ \\
& S3 & $-0,30$ & 1,03 & 1,88 & $<0,001$ \\
\hline
\end{tabular}

Tabela 4

Capacidade Preditiva do Modelo de Regressão Logística para as Versões de 70 e 28 Itens da EFS, para o Pertencimento em Grupo Clínico ou de Universitários

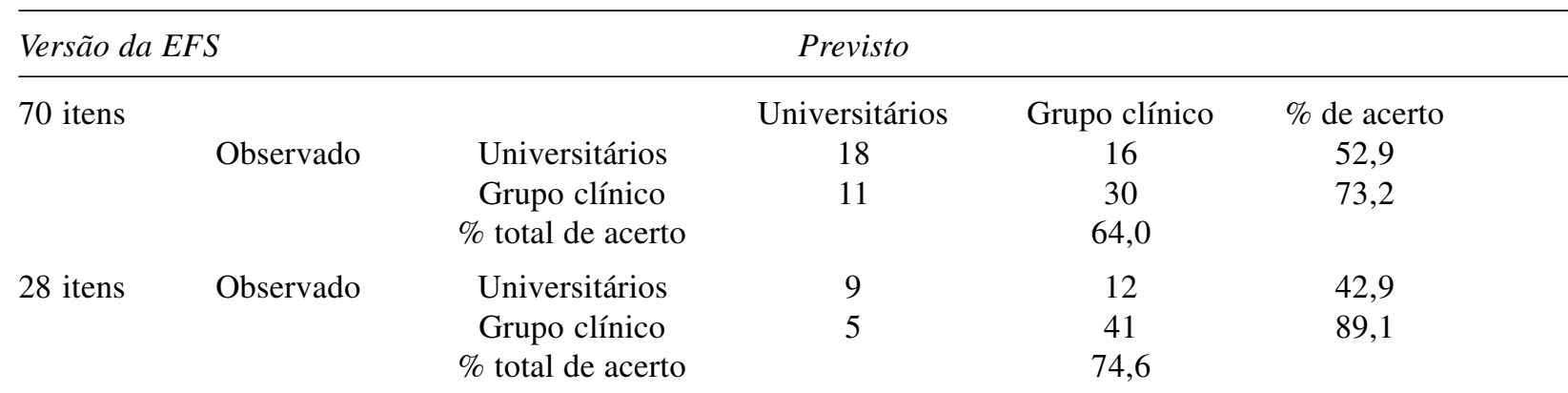


Ao analisar a Tabela 4, é possível verificar que a identificação correta do pertencimento ao grupo de universitários foi melhor com a escala de 70 itens, porém a identificação correta do pertencimento ao grupo clínico foi superior na escala reduzida. De modo geral, a capacidade preditiva do pertencimento a grupos específicos gerado por essa análise evidenciou uma melhora na versão de 28 itens, ou seja, ultrapassou-se a capacidade preditiva geral de $64 \%$ para $74,6 \%$. Desse modo, tanto a análise de diferenças de média do grupo clínico com a amostra normativa como a análise de regressão indicam que as versões da escala apresentam propriedades equivalentes, permitindo a diferenciação de um grupo clínico com a amostra normativa e a manutenção ou melhora da capacidade preditiva de um modelo de regressão que comparou o grupo clínico com universitários.

Outra investigação que verificou a validade de critério da escala original foi feita por Nunes et al. (2006), em que analisaram o uso conjunto da EFS com entrevistas clínicas para a identificação de aspectos associados ao transtorno da personalidade anti-social, considerando a mesma amostra do estudo de Nunes, Nunes, et al. (2009). Nessa pesquisa, além da EFS, os participantes responderam uma entrevista semi-estruturada que buscava detectar indicadores de transtornos da personalidade em contextos variados, como histórico clínico (hisclin), aspectos que favoreciam a manutenção do uso de drogas (manutenção) trajetória escolar (escola), percepção dos avaliadores sobre traços de personalidade que favoreciam o uso de drogas (person), trajetória profissional (trabalho), entre outros. Nesse artigo, os autores calcularam as correlações entre os indicadores obtidos nas entrevistas e os resultados das subescalas da EFS agrupando as duas amostras avaliadas, ou seja, considerando o grupo clínico e o de universitários conjuntamente (Tabela 5). Essa análise foi realizada para verificar se, efetivamente, os resultados obtidos com esses métodos diferentes (entrevista e escala) geravam informações coerentes entre si. São apresentados na Tabela 5 apenas os indicadores da entrevista que apresentaram correlações significativas com as facetas da EFS.

Tabela 5

Correlações entre os Indicadores da Entrevista e EFS para as Versões de 70 e 28 Itens $(N=74)$

\begin{tabular}{|c|c|c|c|c|c|}
\hline Versão da EFS & $\begin{array}{l}\text { Indicadores da entrevistal } \\
\text { Facetas da EFS }\end{array}$ & $S 1$ & $S 2$ & $S 3$ & Escore geral \\
\hline 70 itens & $\begin{array}{l}\text { HISCLIN } \\
\text { MANUTENCAO } \\
\text { PERSON } \\
\text { ESCOLA } \\
\text { TRABALHO }\end{array}$ & $\begin{array}{l}-0,23 * \\
-0,32 * *\end{array}$ & $\begin{array}{l}-0,31^{* *} \\
-0,34^{* *} \\
-0,37^{* *} \\
-0,41^{* *}\end{array}$ & $\begin{array}{l}-0,32^{* *} \\
-0,33^{* *} \\
-0,33^{* *} \\
-0,47^{* *}\end{array}$ & $\begin{array}{l}-0,36 * * \\
-0,30 * * \\
-0,31 * * \\
-0,51 * *\end{array}$ \\
\hline 28 itens & $\begin{array}{l}\text { HISCLIN } \\
\text { MANUTENCAO } \\
\text { PERSON } \\
\text { ESCOLA } \\
\text { TRABALHO }\end{array}$ & $-0,34 * *$ & $\begin{array}{l}-0,47 * * \\
-0,49^{* *} \\
-0,50^{* *} \\
-0,57^{* *} \\
-0,35^{* *}\end{array}$ & $\begin{array}{c}-0,31^{* *} \\
-0,37^{* *} \\
-0,25^{*} \\
-0,47^{* *}\end{array}$ & $\begin{array}{c}-0,45^{* *} \\
-0,40 * * \\
-0,36 * * \\
-0,59 * * \\
-0,24 *\end{array}$ \\
\hline
\end{tabular}

Nota. Legenda: * $p<0,05 ; * * p<0,01$.

Pode-se notar que as correlações encontradas entre as medidas de Socialização e os indicadores levantados por meio das entrevistas clínicas foram maiores para a versão reduzida que para a EFS completa. Analisando as correlações mais detalhadamente, os mesmos indicadores que se relacionaram significativamente com a faceta Amabilidade (S1) o fizeram tanto na versão completa como na reduzida, tendo o mesmo ocorrido com Confiança nas pessoas (S3). No entanto, a faceta Pró-sociabilidade (S2) e o escore geral da EFS apresentaram uma correlação que não havia sido significativa com a versão completa da escala, mas que atingiu o nível de significância mínimo esperado com a versão reduzida, a saber, o indicador associado ao histórico no trabalho. Esses resultados, em conjunto, sugerem que a seleção dos itens para a versão reduzida da EFS foi realizada de forma ade- quada e que houve uma manutenção das propriedades psicométricas da escala, tanto analisados por meio de Rasch como pela reanálise dos estudos de validade de critério.

\section{Considerações Finais}

O construto Socialização tem se mostrado relevante em contextos variados, entre eles, na clínica psicológica, no tratamento de sintomas paranóides, anti-sociais, narcisistas e de personalidades dependentes (Widiger et al., 2002) e no âmbito organizacional, em que é possível destacar comportamentos de maior cooperação e menor competição, além da utilidade na previsão de melhores performances no trabalho (Barrick \& Mount, 1991; Ross et al., 2003; Witt et al., 2002). Nos contextos supra-citados e em outros, há utilidade para a criação de escalas de 
avaliação curtas, do mesmo modo que para a realização de pesquisas para avaliar esse construto.

Considerando a relevância do estudo de instrumentos que avaliem o construto Socialização, os objetivos dessa pesquisa abarcaram a seleção de itens da EFS para a construção de uma versão reduzida da escala e a comparação dos estudos da versão completa com a versão reduzida. Para a seleção de itens, utilizou-se da Análise de Rasch e uma análise qualitativa. A comparação das duas versões da escala foi feita pela Análise Rasch, em que foram analisados os parâmetros theta das pessoas, infit e outfit e a consistência interna, além da reanálise de dois estudos de validade de critério realizados com a EFS.

Por meio das análises psicométricas realizadas, pôdese constatar que as propriedades da escala foram preservadas ao reduzí-la, no que diz respeito ao theta avaliado, ao infit e outfit. Houve uma pequena perda de precisão da escala com a sua redução, resultado esse compreensível uma vez que a fórmula para o cálculo desse parâmetro relaciona de forma direta o valor encontrado e o número de itens. Ainda assim, os índices de consistência interna da versão reduzida da EFS apresentaramse adequados (Pasquali, 2003; Wright \& Stone, 2004). Desse modo, defende-se que os parâmetros psicométricos da versão reduzida são favoráveis à possibilidade de utilização da mesma. De modo semelhante, a reanálise dos estudos de validade de critério da EFS com a versão reduzida confirmou a utilidade da mesma na identificação de características mais marcantes em pessoas com quadro de dependência química, quando comparadas com pessoas da população geral. Ainda, a reanálise dos estudos de validade de critério reforçou a utilidade da versão reduzida para o levantamento de sintomas associados ao transtorno de personalidade anti-social.

A proposta de redução da EFS torna-se relevante pelo tamanho original da escala, que pode limitar seu uso em situações em que o psicólogo dispõe de pouco tempo para a avaliação. Assim, presentemente buscou-se atingir os objetivos destacados por Gosling et al. (2003), no que diz respeito à disponibilização de escalas curtas, para serem utilizadas em situações em não é possível realizar uma avaliação mais extensa. Como proposta para estudos futuros, sugere-se a aplicação da versão reduzida presentemente analisada, de modo a comparar os resultados obtidos com a base de dados da normatização do teste completo com a nova versão.

Outra questão pertinente a ser investigada em trabalhos posteriores se refere a melhor capacidade preditiva da versão reduzida em detectar o grupo clínico do que a versão completa, que foi observado por meio regressão logística. Esse dado é intrigante uma vez que a versão original, diferentemente da reduzida, contempla itens com conteúdo clínicos, o que não foi privilegiado na versão reduzida. Ressalta-se que a amostra em que foi identificado esse resultado é restrita a dependentes químicos, o que reforça a necessidade de explorar a EFS com po- pulações que apresentem outros quadros clínicos, para verificar se esse padrão se sustenta.

\section{Referências}

Allport, G. W. (1961). Pattern and growth in personality. New York: Holt, Rinehart and Winston.

Ávila, L. M., \& Stein, L. M. (2006). A influência do traço de personalidade neuroticismo na suscetibilidade a falsas memórias. Psicologia: Teoria e Pesquisa, 22(3), 339-346.

Baker, F. B. (2001). The basics of Item Theory Response. Washington, DC: ERIC.

Barrick, M. R., \& Mount, M. K. (1991). The Big Five personality dimensions and job performance: A metaanalysis. Personnel Psychology, 44, 1-26.

Bueno, J. M. H., Oliveira, S. M. S. S., \& Oliveira, J. C. S. (2006). Um estudo correlacional entre habilidades sociais e traços de personalidade. PsicoUSF, 6(1), 31-38.

Conselho Federal de Psicologia. (2007). SATEPSI- Lista dos testes aprovados. Retrieved August 20, 2007 from http:// www.pol.org.br

Costa, F. R. (2003). CPS: Escala de Personalidade de Comrey (2. ed.). São Paulo, SP: Vetor Editora Psico-Pedagógica.

Costa, P. T., Jr., \& McCrae, R. R. (1992). Revised NEO Personality Inventory (NEO-PI-R) and Five Factor Inventory (NEO-FFI) professional manual. Odessa, FL: Psychological Assessment Resources.

Costa, P. T., Jr., \& Widiger, T. A. (2002). Introduction. In P. T. Costa \& T. A. Widiger (Eds.), Personality disorders and the Five-Factor Model of Personality (2 ${ }^{\text {nd }}$ ed., pp. 3-16). Whashington, DC: American Psychological Association.

Digman, J. M. (2002). Historical antecedents of the Five-Factor Model. In P. T. Costa \& T. A. Widiger (Eds.), Personality disorders and the Five-Factor Model of Personality $\left(2^{\text {nd }}\right.$ ed., pp. 17-22). Washington, DC: American Psychological Association.

Embretson, S., \& Reise, S. (2000). Item Response Theory for Psychologists. Mahwah, NJ: Lawrence Erlbaum.

Goldberg, L. R. (1982). From ace to zombie: Some exploration in the language of personality. In C. D. Spielberg \& J. N. Butcher (Eds.), Advances in personality assesment (pp. 7389). Hilsdale, NJ: Erlbaun.

Gosling, S. D., Rentfrow, P. J., \& Swann, W. B., Jr. (2003). A very brief measure of the Big Five Personality domain. Journal of Research in Personality, 37, 504-528.

Hutz, C. S., \& Nunes, C. H. S. S. (2001). Escala Fatorial de Neuroticismo. São Paulo, SP: Casa do Psicólogo.

Hutz, C. S., Nunes, C. H. S. S., Silveira, A. D., Serra, J., Anton, M., \& Wieczorek, L. S. (1998). O desenvolvimento de marcadores para a avaliação da personalidade no modelos dos Cinco Grandes Fatores. Psicologia: Reflexão e Crítica, 11, 395-409.

Maciel, M. J. N., \& Yoshida, E. M. P. (2006). Avaliação de alexitimia, neuroticismo e depressão em dependentes de álcool. Avaliação Psicológica, 5(1), 43-54.

McCrae, R. R., \& John, O. P. (1992). An introduction to the Five-Factor Model and its applications. Journal of Personality, 60, 175-216.

Nunes, C. H. S. S. (2005). Construção, normatização e validação das escalas de socialização e extroversão no modelo dos Cinco Grandes Fatores. Tese de Doutorado nãopublicada, Instituto de Psicologia, Universidade Federal do Rio Grande do Sul, Porto Alegre, RS. 
Nunes, C. H. S. S., \& Hutz, C. S. (2007a). Escala Fatorial de Extroversão: Manual técnico. São Paulo, SP: Casa do Psicólogo.

Nunes, C. H. S. S., \& Hutz, C. S. (2007b). Escala Fatorial de Socialização: Manual técnico. São Paulo, SP: Casa do Psicólogo.

Nunes, C. H. S. S., \& Hutz, C. S. (2007c). Construção e validação da Escala Fatorial de Socialização no Modelo dos Cinco Grandes Fatores. Psicologia: Reflexão e Crítica, 20(1), 20-25.

Nunes, C. H. S. S., Hutz, C. S., \& Nunes, M. F. O. (2010). Bateria Fatorial de Personalidade (BFP)- Manual técnico. Itatiba, SP: Casa do Psicólogo.

Nunes, C. H. S. S., Nunes, M. F. O., \& Hutz, C. S. (2006). Uso conjunto de escalas de personalidade e entrevista para a identificação de indicadores de transtorno anti-social. Avaliação Psicológica, 5(2), 171-178.

Nunes, C. H. S. S., Nunes, M. F. O., Cunha, T. F., \& Hutz, C. S. (2009). Relationships between chemical addiction and personality structure in a Brazilian sample. Interamerican Journal of Psychology, 43(1), 68-76.

Nunes, C. H. S. S., Primi, R., Nunes, M. F. O., Nascimento, M. M., Cunha, T. F., \& Couto, G. (2008). Teoria de Resposta ao Item para otimização de escalas tipo Likert: Um exemplo de aplicação. Revista Iberoamerica de Diagnóstico y Evaluación Psicológica, 25(1), 51-79.

Pasquali, L. (2003). Psicometria: Teoria dos testes na Psicologia e na Educação. Petrópolis: RJ: Vozes.

Pasquali, L., Azevedo, M. M., \& Ghesti, I. (1997). Inventário Fatorial de Personalidade-IFP. São Paulo, SP: Casa do Psicólogo.

Pérez, E., Cupani, M., \& Beltramino, C. (2004). Adaptacion del Inventario de Personalidad 16PF-IPIP a un contexto de orientacioìn. Estudio preliminar. Evaluar, 4, 23-47.

Reise, S., \& Henson, J. M. (2000). Computerization and adaptive administration of the NEO PI-R. Assessment, 7(4), 347-364.

Ross, S. R., Rausch, M. K., \& Canada, K. E. (2003). Competition and cooperation in the Five-Factor Model: Individual differences in achievement orientation. The Journal of Psychology, 137(4), 323-337.

Santos, A. A. A., Sisto, F., \& Martins, R. M. M. (2003). Estilos cognitivos e personalidade: Um estudo exploratório de evidências de validade. Psico USF, 8(1), 11-19.

Tupes, E. C., \& Christal, R. E. (1992). Recurrent personality factors based on trait ratings. Journal of Personality, 60, 225-252. (Original work published 1961)

Urbina, S. (2007). Fundamentos da testagem psicológica. Porto Alegre, RS: Artmed.

Widiger, T. A., Trull, T. J., Clarkin, J. F., Sanderson, C., \& Costa, P. T. (2002). A description of the DSM-IV personality disorders with the Five-Factor Model of Personality. In P. T. Costa \& T. A. Widiger (Eds.), Personality disorders and the Five-Factor Model of Personality (2 ${ }^{\text {nd }}$ ed., pp. 89-102). Washington, DC: American Psychological Association.

Witt, L. A., Kacmar, K. M., Carlson, D. S., \& Zivnuska, S. (2002). Interactive effects of personality and organizational politics on contextual performance. Journal of Organizational Behavior, 23(8), 911-926.

Wright, B. D., \& Masters, G. N. (1982). Rating Scale Analysis. Chicago: MESA.
Wright, B. D., \& Stone, M. H. (2004). Making measure. Chicago: The Phaneron Press.

Zacharias, J. J. M. (2003). Questionário de Avaliação Tipológica (QUATI): Versão II: Manual. São Paulo, SP: Vetor. 